\title{
Stabilization of Aqueous Dispersions of Inorganic Microparticles Under Mechanical Activation
}

\author{
A. V. PERChenoK ${ }^{1}$, E. V. SuVOrova ${ }^{1}$, A. A. FARMAKOVSKAYA ${ }^{1}$, V. KOHLERT ${ }^{2}$, \\ ${ }^{1}$ Moscow Aviation Institute (National Research University), \\ Moscow, Volokolamskoe shosse, 4, \\ RUSSIA, \\ ${ }^{2}$ University of Stuttgart, \\ Stuttgart, Pfaffenwaldring, 55, \\ GERMANY
}

\begin{abstract}
The stability of aqueous dispersed systems of $\mathrm{TiO} 2$ and $\mathrm{Fe} 2 \mathrm{O} 3$ microparticles in the presence of a water-soluble polymer - ethylhydroxyethylcellulose (EHEC) - has been studied under the condition of using two methods of mechanoactivation of the pigment surface - processing them in a disintegrator and in an ultrasonic field. It is shown that in the absence of a polymer stabilizer, an intense effect on aqueous dispersed systems of pigments leads to rapid coagulation of particles. In the presence of EHEC, a small stabilizing effect is observed, which is significantly enhanced when processing dispersed systems in a disintegrator and in an ultrasonic field. In this case, the stability of dispersed systems depends little on the method of mechanoactivation of their surface, but significantly depends on the duration of the intense exposure.
\end{abstract}

Keywords_-Pigments, stability, particles, mechanical activation, ultrasound.

Received: November 27, 2020. Revised: August 14, 2021. Accepted: September 1, 2021. Published: September 8, 2021.

\section{Introduction}

T $\mathrm{N}$ recent years, environmentally friendly aqueous dispersions 1 of pigments widely used in the paint and varnish and printing industries have attracted great scientific and practical interest. An important problem for dispersed systems is the problem of their stability. To stabilize dispersions, surfactants are usually used - low molecular weight and polymeric.

A number of works have shown that some water-soluble polymers (such as polyvinyl alcohol, polyvinylpyrrolidone, polyvinylcaprolactam, cellulose derivatives) can be used as stabilizers of aqueous dispersed systems [1]-[4]. In this case, polymers are reversibly and irreversibly sorbed on the surface of solid particles, forming protective adsorption-solvation layers. This method of stabilizing dispersed systems is commonly called adsorption-chemical modification.

Recently, works have appeared showing that, in order to obtain highly dispersed stable systems, it is effective to use, along with adsorption-chemical modification of the surface of a solid phase, mechanical action on it - mechanochemical modification [5]-[18].

In a number of studies, the method of mechanochemical modification (mechanical action during processing in mills of various types) was used to obtain dispersions of oxides of iron, chromium and lead in organic solvents in the presence of polymers [4]-[10]. The authors conclude that the rate of adsorption of the polymer from the solvent on the surface of metal oxides under mechanical action increases due to structural changes in the surface layer of the solid phase.

Mechanical dispersion is a productive way to obtain large quantities of nanopowders of various materials: metals, alloys, intermetallic compounds, ceramics, composites. As a result of mechanical grinding and mechanical fusion, complete solubility in the solid state of elements can be achieved, the mutual solubility of which is negligible under equilibrium conditions [19]-[37]. The most common equipment for the mechanical activation of powders and the implementation of mechanochemical processes are mills (reactors) of various types, in which the value of the specific mechanical energy transferred to the powder can reach $10^{2}-10^{3} \mathrm{~kJ} / \mathrm{g}$.

By mechanical grinding, the size of microcrystalline primary particles can be reduced to $10 \mathrm{~nm}$ or less, which is equivalent to a reduction in the diffusion length required for the reaction [10]. With the joint mechanical processing of several components of the mixture, deformation mixing and mechanochemical reactions occur. The features of lowtemperature mixing of solid reagents caused by the formation of intermediate metastable states and the possibilities of mechanochemical synthesis of various compounds are discussed in detail in [10]-[11].

During mechanical dispersion of solid particles, plastic deformation develops according to the dislocation mechanism and is initially localized in shear bands containing a large number of dislocations. Upon reaching a certain stress level, these dislocations combine and recombine into low-angle boundaries separating individual grains, capturing impurity atoms. At this stage of dispersion, particles with a diameter of 
20-30 nm are formed, and their number increases with abrasion. In ductile metals, grain boundaries are usually formed by the polygonization mechanism [15]. At the next stage of grinding, the orientation of individual crystallites relative to each other becomes random due to sliding along the grain boundaries. This behavior during milling is typical for metals and intermetallic compounds with a bcc lattice [14].

The mechanical action during the dispersion of the material is impulsive; therefore, the stress field does not act during the entire residence time of the particles in the reactor, but only at the moment of their collision. In a short time after the collision, partial relaxation occurs. For this reason, during mechanochemical synthesis, it is necessary to take into account the nature of the formation of the stress field in time and the kinetics of subsequent relaxation processes. Mechanical action is not only impulsive, but also local, since it does not occur in the entire mass of a solid, but only where a stress field arises and then relaxes.

Among the many such approaches, the use of ultrasound for the mechanochemical synthesis of materials has been widely studied for many years and is now positioned as one of the most powerful tools in the synthesis of nanostructured materials [14]-[18].

Almost all processes for the preparation of nanoparticles can be attributed to the number of redox reactions in aqueous solutions, reactions of decomposition (dispersion) of substances during cavitation and partially to reactions in nonaqueous systems, since water acts as the main solvent or reagent in them, although organic substance. Therefore, it is advisable to subdivide the processes of obtaining nanoparticles according to the types of reactions (for example, homogeneous, heterogeneous), and also to classify according to the types of substances and the composition of the resulting nanoparticles. This approach is used in some works [16-20]. The results of the impact of acoustic vibrations on chemical processes are proposed to be divided into the so-called effects of the first and second order. These are nonlinear effects that develop in liquids when powerful acoustic waves propagate. Compared to traditional energy sources, ultrasonic exposure provides rather unusual reaction conditions (short duration of extremely high temperatures and pressures in liquids), which cannot be realized by other methods.

However, with this approach, it is impossible to assess the degree of influence of individual ultrasound parameters on chemical reactions. Nevertheless, the equations describing each of these parameters and effects can be used to formalize the problems of mathematical modeling of the corresponding chemical processes [38]-[56].

The action of acoustic vibrations is very diverse. Ultrasonic waves can accelerate chemical reactions due to emulsification of liquids and their components [28], dispersion of solid components, erosion of their surface, degassing, prevention of precipitation or coagulation of products, intensive mixing, etc. [29]. The action of ultrasound - solids is far from always reduced only to dispersion, since under certain conditions, the activity turns out to be much higher than that which would be expected after a simple grinding of particles. As a result of dispersion, the area of the interface between the phases of the reacting components increases. Dispersion in this case occurs both due to the destruction of particles of the solid phase and due to surface friction between the solid and liquid phases. As a result of a decrease in the thickness of the diffusion boundary layer, the number of effective collisions of molecules of reacting components increases [30]. The process of erosion of solids under the influence of low sound frequencies has the following feature: if, when using medium and high frequencies, rather large particles are crumbled from the surface, as a result of which it becomes rough, then under the influence of low frequencies, smoothing and a kind of polishing occurs. surface, apparently due to the chipping of microscopic particles from it.

In this regard, it is extremely useful to consider methods for synthesizing nanomaterials using ultrasound in order to provide a fundamental understanding of their basic principles and demonstrate the powerful and unique aspects of ultrasound in the synthesis of nanostructured materials.

High-intensity ultrasound has found many applications in organic synthesis, materials science and organometallic chemistry, as well as in industrial manufacturing processes. The use of high-intensity ultrasound provides a simple and versatile tool for the synthesis of nanostructured materials that are often unavailable by conventional methods. The main physical phenomena associated with ultrasound that are relevant to the synthesis of nanomaterials are cavitation and sputtering. Acoustic cavitation (formation, growth, and instantaneous collapse of bubbles in a liquid) creates extreme conditions inside a collapsing bubble and is the source of most sonochemical phenomena in liquids or liquid-solid suspensions. Another option is atomization (the creation of fog due to the passage of ultrasound through the liquid and hitting the liquid-gas interface), which is the basis of ultrasonic spray pyrolysis (USP) with subsequent reactions occurring in heated mist droplets. Sonochemical phenomena arise from extreme transient conditions caused by ultrasound, which creates unique conditions that can reach temperatures in excess of $5000 \mathrm{~K}$, pressures in excess of 1000 atmospheres, and heating and cooling rates in excess of $1010 \mathrm{~K} / \mathrm{s}$.

The speed of sound in a typical liquid is from 1000 to 1500 $\mathrm{m} / \mathrm{s}$, and the ultrasonic wavelengths can vary from about $10 \mathrm{~cm}$ to $100 \mu \mathrm{m}$ in the frequency range from $20 \mathrm{kHz}$ to $15 \mathrm{MHz}$, which is much larger than the characteristic sizes of molecules. Thus, the chemical and physical effects of ultrasound do not arise from the direct interaction between chemicals and sound waves, but rather from the physical phenomenon of acoustic cavitation: the formation, growth and collapse of cavitation microbubbles. When longitudinal acoustic vibrations of a certain amplitude propagate through the liquid, the liquid is under dynamic tensile stress, and the density changes with alternating waves of expansion and contraction. Discontinuities in the medium (bubbles) are often formed from 
pre-existing impurities (for example, gas-filled gaps in dust grains) and vibrate with the applied sound field. Bubbles can grow by slowly pumping gas out of the liquid volume into an oscillating bubble (rectified diffusion). Bubbles of a critical size (usually tens of micrometers) can strongly influence the acoustic field and undergo rapid inertial growth during expansion followed by instantaneous collapse. The bubble collapse process is almost adiabatic in its final stages and is the cause of the extreme conditions characteristic of sonochemistry.

There are two main approaches to explaining the energyintensive chemical and physicochemical effects caused by cavitation: thermal and electrical. These effects were thoroughly studied by several groups of researchers [2]-[9]. With the thermal approach, these effects are associated with a high temperature inside the cavitation bubble, achieved during its adiabatic compression with a continuously increasing rate, and with an electric approach, with a discharge inside the bubble due to the accumulation of electric charges on its walls.

It was previously shown that treatment in an ultrasonic field of aqueous dispersions of titanium dioxide in the presence of water-soluble polymers leads to a significant increase in their stability [5]-[18]. It is assumed that in the process of mechanical activation in the presence of a polymer stabilizer, two processes occur simultaneously: the disaggregation of pigment agglomerates and the formation of protective adsorption-solvation polymer layers on the "freshly formed" and therefore "active" surface of the solid phase. As a result, highly dispersed stable systems are obtained.

However, these assumptions have not yet found experimental confirmation and the mechanism of mechanoactivation processes remains unclear [2]-[9].

In this work, the task was set to obtain highly dispersed stable aqueous systems of $\mathrm{TiO}_{2}$ and $\mathrm{Fe}_{2} \mathrm{O}_{3}$ pigments in the presence of the EHEC polymer stabilizer as a result of mechanical action on them in two ways - by treating them in an ultrasonic field and in a disintegrator.

\section{Experimental part}

\subsection{Materials}

Pigment $\mathrm{Fe}_{2} \mathrm{O}_{3}$ from Bayferrox; the surface of the pigment is treated with aluminum and silicon oxides.

Pigment $\mathrm{TiO}_{2} \mathrm{RN}-56$ from Kronos; the surface is treated with oxides of aluminum, silicon and zirconium to reduce light sensitivity.

A non-toxic water-soluble polymer, ethyl hydroxyethyl cellulose (EHEC) with a molecular weight of 60,000 , was used as a stabilizer for aqueous dispersions of pigments.

\subsection{Methods}

For study the dispersed systems, standard methods were applied, as it has been already described in our previous studies [2]-[9].
The sedimentation behavior of dispersed systems was investigated as follows: a weighed portion of the pigment was mixed with an aqueous dispersion medium. The dispersion was placed in a glass cylinder with graduations and, then, the descent of the interface between the solid phase and the pure dispersion medium was monitored in time. Sedimentation curves were plotted as the dependence of the sedimentation volume $\mathrm{V}_{\text {sed. }}, \mathrm{ml}$. from the time of sedimentation (min., logarithmic coordinates).

The average particle size of fillers and pigments was evaluated using a Photocor Compact device.

Mechanical activation by the ultrasound method was carried out on an UZDN-2 ultrasonic generator with immersed sonotrode with a frequency of $22 \mathrm{kHz}$ and an intensity of 30 $\mathrm{W} / \mathrm{cm}^{2}$; the treatment time was varied from 2 to $30 \mathrm{~min}$.

For mechanical activation, we also used a laboratory disintegrator manufactured by the Disintegrator company (Estonia), of a through-type type, containing five rows of discs with teeth that rotate towards each other at a speed of about 1000 revolutions per minute.

\section{Results and discussion}

It is important to emphasize that in the studies, pigments were used, the surface of which was treated with oxides of aluminum, silicon and zirconium. This circumstance suggests that the properties of the studied dispersions of $\mathrm{TiO}_{2}$ and $\mathrm{Fe}_{2} \mathrm{O}_{3}$ pigments are similar.

The results of studying the effect of mechanical activation (ultrasonic treatment and disintegrator treatment (DI treatment) of aqueous dispersions of $\mathrm{TiO}_{2}$ pigment in the presence of EHEC on their sedimentation stability (Fig. 1), as well as on the average particle size of the corresponding dispersions show that in the absence of a polymer, dispersions are treated in an ultrasonic field and DI leads to their rapid coagulation, and the particle size is on average $1,0-1,1 \mu \mathrm{m}$. This is apparently due to the formation of a freshly formed surface with uncompensated charges on the $\mathrm{TiO}_{2}$ surface during mechanical activation. The system tends to reduce the surface energy, which leads to a sharp decrease in the stability of dispersions.

The presence of EHEC in the system slightly increases the stability of dispersions, and the particle size decreases to 0,5 $\mu \mathrm{m}$. With mechanical activation (ultrasonic or DI treatment) in the presence of EHEC, the stability of dispersions increases significantly (approximately by a factor of 100). The particle size is thereby reduced to the original particle size of the titanium dioxide used.

A similar effect can be observed by analyzing the sedimentation stability of $\mathrm{Fe}_{2} \mathrm{O}_{3}$ pigment dispersions in water and in an EHEC solution, and the data on the particle sizes confirm a significant decrease in the particle size of the dispersions after their ultrasonic and DI treatment.

These results are presented in the Fig. 1 and 2. 


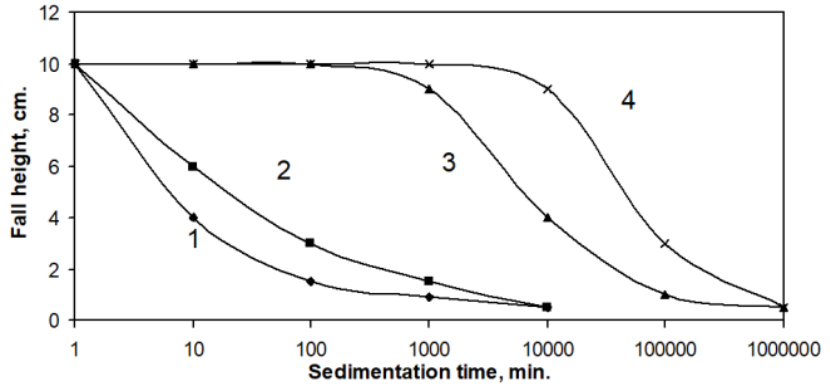

Fig. 1. Sedimentation curves for $\mathrm{TiO}_{2}$ pigment in absence and in presence of EHEC.

1 - without EHEC, ultrasonic treatment 2 min.,

2 - without EHEC, no ultrasonic treatment,

3 - with EHEC, no ultrasonic treatment,

4 - with EHEC, ultrasonic treatment 2 min.

Concentration of $\mathrm{TiO}_{2}$ and $\mathrm{EHEC}-1 \%$ wt.

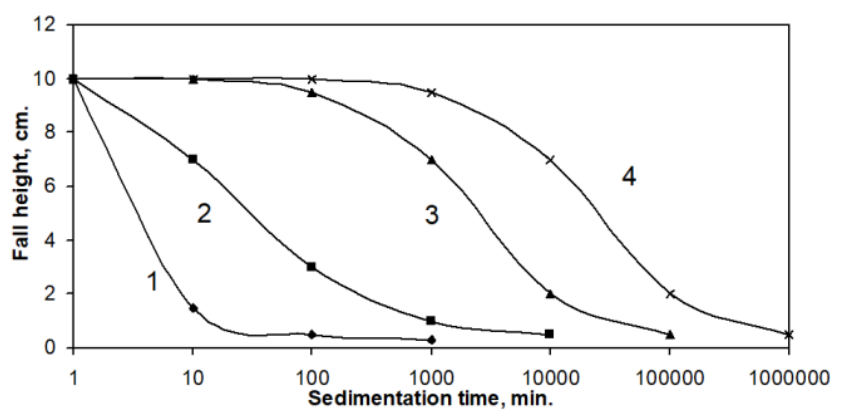

Fig. 2. Sedimentation curves for $\mathrm{Fe}_{2} \mathrm{O}_{3}$ pigment in absence and in presence of EHEC.

1 - without EHEC, ultrasonic treatment 2 min.,

2 - without EHEC, no ultrasonic treatment,

3 - with EHEC, no ultrasonic treatment,

4 - with EHEC, ultrasonic treatment 2 min.

Concentration of $\mathrm{Fe}_{2} \mathrm{O}_{3}$ and EHEC - 1\% wt.

It should be noted that the stability of systems obtained as a result of processing in an ultrasonic field is somewhat higher than the stability of the same dispersions after their DI processing, but this difference is insignificant.

Thus, common to the investigated aqueous dispersions of inorganic pigments $\mathrm{TiO}_{2}$ and $\mathrm{Fe}_{2} \mathrm{O}_{3}$ is the disaggregation of pigment particles during mechanoactivation, and in the presence of a polymer stabilizer, protective adsorptionsolvation layers are formed, which, apparently, is the reason for the high activity of mechanoactivation methods for increasing the stability of dispersed systems.

However, it should be noted that polymers can degrade during mechanical activation. This fact can significantly affect the efficiency of the method of mechanochemical modification.

In fact, when studying the sedimentation of dispersions of the pigment $\mathrm{Fe}_{2} \mathrm{O}_{3}$ in an aqueous solution of EHEC at different durations of ultrasonic treatment, it was found that the optimal processing time for obtaining stable dispersions of this pigment is $2 \mathrm{~min}$. An increase in the time of ultrasound exposure leads to a significant decrease in the stability of systems. It can be assumed that EHEC, being an insufficiently flexible polymer, undergoes mechanical destruction, which weakens its stabilizing effect.

The experimental results showing the particle size distribution of aqueous dispersions of the $\mathrm{Fe}_{2} \mathrm{O}_{3}$ pigment in the presence of EHEC before and after ultrasonic treatment show that the distribution curve narrows significantly upon ultrasonic treatment of the dispersion, which indicates the obtaining of a $\mathrm{Fe}_{2} \mathrm{O}_{3}$ dispersion with a more homogeneous structure.

The power of the physical effects of high-intensity ultrasound is well illustrated by the mechanochemical reactions of polymers, which include cavitation-induced rupture of covalent bonds. It has been well known that ultrasonic irradiation of solutions of high molecular weight polymers (both biopolymers and synthetic) leads to chain rupture and the formation of macroradicals [56-58]. Sonication accelerates rearrangement reactions, shifting towards reaction mechanisms that produce molecules that cannot be produced by purely thermal or light-induced reactions. Thus, the mechanical force induced by ultrasound can change the shape of the potential energy curves of chemical reactions, so that reactions that are otherwise impossible can proceed under milder conditions. Ultrasound makes it possible to implement reaction mechanisms that can differ sharply from those achieved by a simple change in chemical or physical parameters. If sensitive to mechanical stress and shock waves and cleavable groups are integrated into the polymer chain, sonication can lead to a point breaking of the polymer chains. Some weak covalent bonds, such as peroxide and azo bonds, tense cycles, and weak coordination bonds (for example, palladium-phosphorus bonds), can be broken by ultrasound.

Ultrasonic treatment can also optimize the process of sol-gel synthesis, a universal method for the preparation of nanostructured metal oxides. The use of ultrasound in the solgel synthesis of nanoparticles can accelerate the hydrolysis process to obtain nanoparticles of metal oxides with a narrower size distribution, larger surface area, and improved phase purity. A wide variety of nanosized metal oxides have been obtained by sonochemical methods. A number of works report the synthesis and stabilization of various nanostructured metal oxides, including $\mathrm{TiO}_{2}, \mathrm{Mn}_{3} \mathrm{O}_{4}, \mathrm{ZnO}, \mathrm{ZrO}_{2}$ [5]-[18]. In these synthesis processes, sonication of aqueous solutions of metal salts is carried out under ambient conditions (usually in the presence of air) to obtain various forms of nanostructured metal oxides. For example, nanometer-sized $\alpha$-nickel hydroxide was prepared simply by sonicating an aqueous solution of nickel nitrate and urea. It is believed that ultrasonic irradiation significantly increases the rate of hydrolysis, and shock waves can cause unusual morphological changes in metal oxides.

For example, in a number of previous works, $\mathrm{TiO}_{2}, \mathrm{ZnO}$, $\mathrm{CeO}_{2}, \mathrm{MoO}_{3}, \mathrm{~V}_{2} \mathrm{O}_{5}$, and $\mathrm{In}_{2} \mathrm{O}_{3}$ nanoparticles were obtained by ultrasonic treatment of their respective precursor solutions [3]- 
[4]. $\mathrm{TiO}_{2}$ nanoparticles obtained by ultrasonic irradiation of a precursor solution are photocatalytically more active than those obtained by the traditional method or commercial $\mathrm{TiO}_{2}$ nanoparticles (for example, Degussa P25). The increase in photocatalytic activity is explained by the improved crystallinity of $\mathrm{TiO}_{2}$, which is achieved through accelerated hydrolysis under the action of ultrasound. Ultrasound can also induce the formation of a unique surface morphology during the synthesis of nanostructured metal oxides in the presence of soft matrices. For example, hollow spinels from $\mathrm{PbWO}_{4}$ were obtained by sonication of a solution of $\mathrm{Pb}\left(\mathrm{CH}_{3} \mathrm{COO}\right)_{2}$, $\mathrm{NaWO}_{4}$ and Pluronic $\mathrm{EO}_{20} \mathrm{PO}_{70} \mathrm{EO}_{20},(\mathrm{Mn}=5800)$.

\section{Conclusion}

It was shown that ultrasonic treatment of aqueous dispersions of inorganic pigments leads to a significant narrowing of the particle size distribution curve, which indicates the obtaining of a dispersion with a more homogeneous structure. The spectral studies carried out allowed us to conclude that EHEC is strongly adsorbed on the surface of pigments, and intense exposure leads to the activation of the processes of adsorption of polymer molecules on the freshly formed surface of pigments. This study opens the prospective for application of water-soluble polymers for synthesis of stable aqueous dispersions of hydrophilic particles.

\section{References}

[1] L. N. Rabinskiy, S. A. Sitnikov, "Development of technologies for obtaining composite material based on silicone binder for its further use in space electric rocket engines," Periodico Tche Quimica, 15 (Special Issue 1), pp. 390-395, 2018.

[2] N. A. Bulychev, A. V. Ivanov, "Effect of vibration on structure and properties of polymeric membranes," International Journal of Nanotechnology, vol. 16, nos. 6/7/8/9/10, pp. $334-343,2019$.

[3] N. A. Bulychev, A. V. Ivanov, "Nanostructure of OrganicInorganic Composite Materials Based on Polymer Hydrogels," International Journal of Nanotechnology, vol. 16, nos. 6/7/8/9/10, pp. $344-355,2019$.

[4] N. A. Bulychev, A. V. Ivanov, "Study of Nanostructure of Polymer Adsorption Layers on the Particles Surface of Titanium Dioxide," International Journal of Nanotechnology, vol. 16, nos. 6/7/8/9/10, pp. $356-365$, 2019.

[5] O. A. Butusova, "Surface Modification of Titanium Dioxide Microparticles Under Ultrasonic Treatment," International Journal of Pharmaceutical Research, vol. 12, i. 4, pp. 2292-2296, 2020.

[6] O. A. Butusova, "Stabilization of Carbon Microparticles by High-Molecular Surfactants," International Journal of Pharmaceutical Research, vol. 12, Supplementary Issue 2, pp. 1147-1151, 2020.

[7] Yu. V. Ioni, A. Ethiraj, "New Tailor-Made Polymer Stabilizers for Aqueous Dispersions of Hydrophobic
Carbon Nanoparticles," International Journal of Pharmaceutical Research, vol. 12, i. 4, pp. 3443-3446, 2020.

[8] Yu. V. Ioni, "Nanoparticles of noble metals on the surface of graphene flakes," Periodico Tche Quimica, vol. 17, no. 36, pp. 1199-1211, 2020.

[9] O. A. Butusova, "Vinyl Ether Copolymers as Stabilizers of Carbon Black Suspensions," International Journal of Pharmaceutical Research, vol. 12, Supplementary Issue 2, pp. 1152-1155, 2020.

[10] M. O. Kaptakov, "Catalytic Desulfuration of Oil Products under Ultrasonic Treatment," International Journal of Pharmaceutical Research, vol. 12, Supplementary Issue 2, pp. 1838-1843, 2020.

[11]B. A. Garibyan, "Enhancement of Mechanical Properties of Inorganic Glass under Ultrasonic Treatment," International Journal of Pharmaceutical Research, vol. 12, Supplementary Issue 2, pp. 1829-1832, 2020.

[12]M. O. Kaptakov, "Enhancement of Quality of Oil Products under Ultrasonic Treatment," International Journal of Pharmaceutical Research, vol. 12, Supplementary Issue 2, pp. 1851-1855, 2020.

[13]O. A. Butusova, "Adsorption Behaviour of Ethylhydroxyethyl Cellulose on the Surface of Microparticles of Titanium and Ferrous Oxides," International Journal of Pharmaceutical Research, vol. 12, Supplementary Issue 2, pp. 1156-1159, 2020.

[14]B. A. Garibyan, "Mechanical Properties of Electroconductive Ceramics," International Journal of Pharmaceutical Research, vol. 12, Supplementary Issue 2, pp. 1825-1828, 2020.

[15]M. O. Kaptakov, "Effect of Ultrasonic Treatment on Stability of $\mathrm{TiO}_{2}$ Aqueous Dispersions in Presence of Water-Soluble Polymers," International Journal of Pharmaceutical Research, vol. 12, Supplementary Issue 2, pp. 1821-1824, 2020.

[16] Yu. V. Ioni, "Synthesis of Metal Oxide Nanoparticles and Formation of Nanostructured Layers on Surfaces under Ultrasonic Vibrations," International Journal of Pharmaceutical Research, vol. 12, i. 4, pp. 3432-3435, 2020.

[17] Yu. V. Ioni, A. Ethiraj, "Study of Microparticles Surface Modification by Electrokinetic Potential Measuring," International Journal of Pharmaceutical Research, vol. 12, i. 4, pp. 3436-3439, 2020.

[18] Yu. V. Ioni, "Effect of Ultrasonic Treatment on Properties of Aqueous Dispersions of Inorganic and Organic Particles in Presence of Water-Soluble Polymers," International Journal of Pharmaceutical Research, vol. 12, i. 4, pp. 3440-3442, 2020.

[19] A. N. Astapov, E. L. Kuznetsova, L. N. Rabinskiy, "Operating capacity of anti-oxidizing coating in hypersonic flows of air plasma," Surface Review and Letters, 26(2), pp. 1850145, 2019.

[20]L. N. Rabinskiy, O. V. Tushavina, E. I. Starovoitov, "Study of thermal effects of electromagnetic radiation on the environment from space rocket activity," INCAS Bulletin, 12 (Special Issue), pp. 141-148, 2020. 
[21] O. A. Pashkov, "Influence of Polymer Coatings on the Mechanical Properties of Steel Samples in Tensile and Bending Tests," Turkish Journal of Computer and Mathematics Education (TURCOMAT), vol. 12, no. 5, pp. 542-548, 2021.

[22] O. A. Pashkov, "Investigation of the Effect of Steel Plate Size and Elevated Temperature on Critical Load in Stability Tests," Turkish Journal of Computer and Mathematics Education (TURCOMAT), vol. 12, no. 10, pp. 1657-1663, 2021.

[23] O. A. Pashkov, "Theoretical calculation of the thickness of interphase zones in the Al-A12O3 composite," Turkish Journal of Computer and Mathematics Education (TURCOMAT), vol. 12, no. 10, pp. 1672-1677, 2021.

[24] O. A. Pashkov, "Experimental and Theoretical Study of Mechanical Properties of Matrix Composite Materials," Turkish Journal of Computer and Mathematics Education (TURCOMAT), vol. 12, no. 10, pp. 1678-1684, 2021.

[25]I. P. Lifanov, A. N. Astapov, V. S. Terentieva, "Deposition of heat-resistant coatings based on the $\mathrm{ZrSi} 2-$ MoSi2-ZrB2 system for protection of non-metallic composite materials in high-speed high-enthalpy gas flows," Journal of Physics: Conference Series, vol. 1713, no. 1, pp. 012025, 2020.

[26]I. P. Lifanov, A. A. Yurishcheva, A. N. Astapov, "Hightemperature protective coatings on carbon composites," Russian Engineering Research, vol. 39, no. 9, pp. $804-$ 808, 2019.

[27] A. N. Astapov, I. P. Lifanov, M. V. Prokofiev, "Hightemperature interaction in the $\mathrm{ZrSi} 2-\mathrm{ZrSiO} 4$ system and its mechanism," Russian Metallurgy (Metally), no. 6, pp. $640-646,2019$.

[28] N. A. Bulychev, E. L. Kuznetsova, V. V. Bodryshev, L. N., "Nanotechnological aspects of temperature-dependent decomposition of polymer solutions," Nanoscience and Technology: An International Journal, vol. 9 (2), pp. 9197, 2018.

[29] L. N. Rabinskiy, O. V. Tushavina, V. F. Formalev, "Mathematical modeling of heat and mass transfer in shock layer on dimmed bodies at aerodynamic heating of aircraft," Asia Life Sciences, (2), pp. 897-911, 2019.

[30]B. A. Antufev, O. V. Egorova, L. N. Rabinskiy, "Quasistatic stability of a ribbed shell interacting with moving load," INCAS Bulletin, 11, pp. 33-39, 2019.

[31] O. A. Butusova, "Design and Properties of Magnetically Controlled Sorbents," Turkish Journal of Computer and Mathematics Education (TURCOMAT), vol. 12, no. 5, pp. 515-519, 2021.

[32] O. A. Butusova, "Application of Magnetically Controlled Sorbents for Detoxication," Turkish Journal of Computer and Mathematics Education (TURCOMAT), vol. 12, no. 5, pp. 520-524, 2021.

[33] V. V. Bodryshev, A. V. Babaytsev, L. N. Rabinskiy, "Investigation of processes of deformation of plastic materials with the help of digital image processing," Periodico Tche Quimica, 16(33), pp. 865-876, 2019.

[34] L. N. Rabinskiy, E. L. Kuznetsova, "Analytical and numerical study of heat and mass transfer in composite materials on the basis of the solution of a stefan-type problem," Periodico Tche Quimica, 15 (Special Issue 1), pp. 339-347, 2018.

[35] N. A. Bulychev, L. N. Rabinskiy, "Ceramic nanostructures obtained by acoustoplasma technique," Nanoscience and Technology: An International Journal, 10 (3), pp. 279286, 2019.

[36]N. A. Bulychev, L. N. Rabinskiy, O. V. Tushavina, "Effect of intense mechanical vibration of ultrasonic frequency on thermal unstable low-temperature plasma," Nanoscience and Technology: An International Journal, 11 (1), pp. 15-21, 2020.

[37]A. V. Babaytsev, A. A. Orekhov, L. N. Rabinskiy, "Properties and microstructure of AlSi10Mg samples obtained by selective laser melting," Nanoscience and Technology: An International Journal, 11(3), pp. 213$222,2020$.

[38]N. A. Kucheva, "Investigation of the mechanical properties of heat-protective highly porous composite materials using the effective medium model," Turkish Journal of Computer and Mathematics Education (TURCOMAT), vol. 12, no. 10, pp. 1613-1621, 2021.

[39] O. V. Egorova, Y. K. Kyaw, "Solution of inverse nonstationary boundary value problems of diffraction of plane pressure wave on convex surfaces based on analytical solution," Journal of Applied Engineering Science, 18(4), pp. 676-680, 2020.

[40]A. N. Tarasova, "Vibration-based Method for Mechanochemical Coating Metallic Surfaces," International Journal of Pharmaceutical Research, vol. 12, Supplementary Issue 2, pp. 1160-1168, 2020.

[41] A.V. Babaytsev, E. L. Kuznetsova, L. N. Rabinskiy, O. V. Tushavina, "Investigation of permanent strains in nanomodified composites after molding at elevated temperatures," Periodico Tche Quimica, 17(34), pp. 1055-1067, 2020.

[42] V.N. Dobryanskiy, L. N. Rabinskiy, O. V. Tushavina. "Experimental finding of fracture toughness characteristics and theoretical modeling of crack propagation processes in carbon fiber samples under conditions of additive production," Periodico Tche Quimica, 16(33), p. 325-3366 2019.

[43] L. N. Rabinskiy, O.V. Tushavina. "Problems of land reclamation and heat protection of biological objects against contamination by the aviation and rocket launch site," Journal of Environmental Management and Tourism, 10(5), pp. 967-973, 2019.

[44] V. N. Dobryanskiy, L. N. Rabinskiy, O.V. Tushavina, "Validation of methodology for modeling effects of loss of stability in thin-walled parts manufactured using SLM technology, "Periodico Tche Quimica, 16(33), pp. 650656, 2019.

[45]A. N. Tarasova, "Effect of Reagent Concentrations on Equilibria in Water-Soluble Complexes," International Journal of Pharmaceutical Research, vol. 12, Supplementary Issue 2, pp. 1169-1172, 2020.

[46]A. N. Tarasova, "Effect of Vibration on Physical Properties of Polymeric Latexes," International Journal of 
Pharmaceutical Research, vol. 12, Supplementary Issue 2, pp. 1173-1180, 2020.

[47] V. F. Formalev, É. M. Kartashov, S. A. Kolesnik, "On the Dynamics of Motion and Reflection of Temperature Solitons in Wave Heat Transfer in Limited Regions," Journal of Engineering Physics and Thermophysics, 93(1), pp. 10-15, 2020.

[48]M. O. Kaptakov, "Effect of Thin Polymer Layers on Mechanical Properties of Metal Surfaces," Turkish Journal of Computer and Mathematics Education (TURCOMAT), vol. 12, no. 5, pp. 525-529, 2021.

[49] B. A. Garibyan, "Determination of the Elastic Modulus of the Coating Using a Spherical Indenter," Turkish Journal of Computer and Mathematics Education (TURCOMAT), vol. 12, no. 10, pp. 1594-1600, 2021.

[50]M. O. Kaptakov, "Modelling of Mechanical Properties of Metal Plates with Polymer Coatings," Turkish Journal of Computer and Mathematics Education (TURCOMAT), vol. 12, no. 5, pp. 530-534, 2021.

[51] B. A. Garibyan, "Theoretical Estimations of Influence of Polymer Coatings on the Elastic Modulus and Ultimate Strength of Steel Samples," Turkish Journal of Computer and Mathematics Education (TURCOMAT), vol. 12, no. 10, pp. 1651-1656, 2021.

[52]M. O. Kaptakov, "Investigation of Effective Mechanical Characteristics of Nanomodified Carbon-Epoxide Composite by Numerical and Analytical Methods," Turkish Journal of Computer and Mathematics Education (TURCOMAT), vol. 12, no. 5, pp. 535-541, 2021.

[53]M. O. Kaptakov, "Obtaining of Carbon Fibers Based Composite Materials and Study of Their Mechanical Properties," Turkish Journal of Computer and Mathematics Education (TURCOMAT), vol. 12, no. 10, pp. 1601-1605, 2021.

[54] V. F. Formalev, N. A. Bulychev, E. L Kuznetsova, S. A. Kolesnik, "The Thermal State of a Packet of Cooled Microrocket Gas-Dynamic Lasers," Technical Physics Letters, 46(3), pp. 245-248, 2020.

[55]L. N. Rabinskii, O. V. Tushavina, "Composite Heat Shields in Intense Energy Fluxes with Diffusion," Russian Engineering Research, 39(9), pp. 800-803, 2019.

[56] L. N. Rabinskiy, O. V. Tushavina, "Investigation of the influence of thermal and climate effects on the performance of tiled thermal protection of spacecraft," Periodico Tche Quimica, 16(33), pp. 657-667, 2019.

\section{Creative Commons Attribution License 4.0 (Attribution 4.0 International, CC BY 4.0)}

This article is published under the terms of the Creative Commons Attribution License 4.0 https://creativecommons.org/licenses/by/4.0/deed.en US 